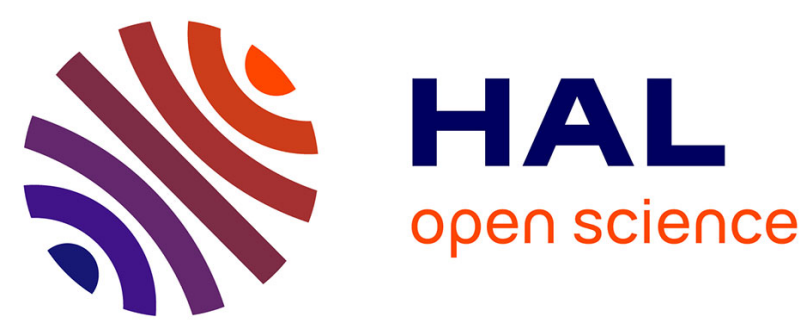

\title{
Fabrication of micro-cages and caged tumor spheroids for microfluidic chip- based assays
}

\author{
Yong He, Boxin Huang, Elrade Rofaani, Jie Hu, Yuanhui Liu, Gabriele \\ Pitingolo, Li Wang, Jian Shi, Carole Aimé, Yong Chen
}

\section{To cite this version:}

Yong He, Boxin Huang, Elrade Rofaani, Jie Hu, Yuanhui Liu, et al.. Fabrication of micro-cages and caged tumor spheroids for microfluidic chip- based assays. Microelectronic Engineering, 2020, 225, pp.111256. 10.1016/j.mee.2020.111256 . hal-02565343v2

\section{HAL Id: hal-02565343 \\ https://hal.sorbonne-universite.fr/hal-02565343v2}

Submitted on 10 Dec 2020

HAL is a multi-disciplinary open access archive for the deposit and dissemination of scientific research documents, whether they are published or not. The documents may come from teaching and research institutions in France or abroad, or from public or private research centers.
L'archive ouverte pluridisciplinaire HAL, est destinée au dépôt et à la diffusion de documents scientifiques de niveau recherche, publiés ou non, émanant des établissements d'enseignement et de recherche français ou étrangers, des laboratoires publics ou privés. 


\title{
Fabrication of micro-cages and caged tumor spheroids for microfluidic chip-based assays
}

\author{
Yong $\mathrm{He}^{\mathrm{a}}$, Boxin Huanga ${ }^{\mathrm{a}}$, Elrade Rofaani ${ }^{\mathrm{a}}$, Jie Hu${ }^{\mathrm{a}}$, Yuanhui Liu ${ }^{\mathrm{a}}$, Gabriele Pitingolo \\ Li Wang ${ }^{\mathrm{b}}$, Jian Shi ${ }^{\mathrm{b}}$, Carole Aiméa and Yong Chen ${ }^{\mathrm{a}^{*}}$ \\ a PASTEUR, Département de chimie, École normale supérieure, PSL University, \\ Sorbonne Université, CNRS, 75005 Paris, France \\ ${ }^{b}$ MesoBioTech, 231 Rue Saint-Honoré, 75001 Paris, France \\ e-mail:yong.chen@ens.fr
}

\begin{abstract}
:
We developed a simple method to fabricate micro-cages and caged tumor spheroids for microfluidic chip-based assays. The micro-cage device consists of an array of honeycomb compartments with a monolayer of cross-linked and agarose-coated gelatin nanofibers at the bottom and a mesh of $200 \mu \mathrm{m}$ hole-size on the top. U87-MG single cells were dispersed through the mesh and resulted tumor spheroids confined in each of the cage compartment after incubation. As expected, the tumor spheroids are one-by-one distributed in each of the compartment with the same size and they grew inside the compartments. The final size of the spheroid was limited by both diffusion and confinement. If the height of the cage is small, the nanofiber layer underneath tumors could be deflected due to mechanic stress of growing tumors. If the height of the cage is large, tumors grew freely without stress but their size was limited by diffusion. In both cases, tumors tended to remain in spherical shape. To illustrate the robustness of the approach, the tumor caged device was reversibly integrated into a microfluidic chip for drug test. Our results show that under tangent flow conditions, combretastatin A-4 had a clear effect on tumor disassembling.
\end{abstract}

Keywords: Tumor spheroid, Microfluidics, Cage device 


\section{Introduction}

Despite decades of intensive research, cancer remains one of the worldwide leading causes of mortality and more important efforts are expected, including both in-vivo and in-vitro assays [1-5]. In this regard, robustly producing and handling of tumor spheroids using cancer cell lines, dissociated cancer cell and cutting tumor samples are necessary for detailed analysis. Previously, Hanging-Drop method $[6,7]$ and Liquid-Overlay method [8-11] were widely used for spheroid formation. These methods are simple, but the resulted spheroids have to be transferred to another culture platform. Rotating bioreactor $[12,13]$ and magnetic levitation $[14,15]$ are also frequently used to increase the production yield but the fabricated spheroids generally lack the size uniformity. More recently, patterned micro-well arrays [1618] were used to improve the uniformity of the tumor spheroids but the applications of these methods are limited since they are too simple to take into account the complexity of the tumor niche, which is a specific microenvironment where tumors are formed and regulated.

To overcome the above limitations, we developed a cage method which allows not only the formation of uniform tumor spheroids but also the integration of the fabricated spheroids into a microfluidic device for different assays. Our cage device is in the form of a patch with honeycomb frame sandwiched by a monolayer of agarose coated nanofibers and a mesh of relatively large openings. Both frame and mesh were defined by UV-lithography and soft-lithography and the nanofibers were produced by electrospinning, in a similar way of culture patch fabrication [19-21]. Single tumor cells could be dispersed through the mesh holes into each of the honeycomb compartments. Upon incubation, cells aggregated to form single spheroids in each of the compartments (Fig. 1). Since the size of the spheroids is generally larger than that of the mesh holes, they could be efficiently caged, allowing robust handling and manipulation. While both mesh and nanofiber monolayer are highly permeable and the patch can be quasi suspended in the culture medium, the spheroid growth conditions should be better than other culture platforms. We thus demonstrate the formation of uniform tumor spheroids and their integration into a microfluidic chip. Interestingly, we observed a significant deflection of the fiber layer due to mechanical forces of the growing spheroids. We also performed a preliminary drug test under both conventional and microfluidic culture conditions. Other types of stresses such as hypoxia could also be applied and the cage device could operate with other devices such as microfilters [22], therefore holding high potential of this approach.

\section{Materials and methods}

\subsection{Fabrication of micro-cage device}

The cage device was fabricated by photolithography, soft-lithography and electrospinning. Firstly, a double layer SU-8 mold was fabricated by photolithography. The mesh layer was patterned on a silicon wafer using a $50 \mu \mathrm{m}$ thick SU-8 negative photoresist (Fig. 2a). Then, the honeycomb frame of height 
in the range of 200 to $300 \mu \mathrm{m}$ was directly patterned on the mesh layer (Fig. 2b). This double layer SU8 mold was then exposed in trimethylchlorosilane (TMCS, Sigma, France) vapor during $10 \mathrm{~min}$ for anti-sticking surface treatment. Afterward, a mixture of PDMS (GE RTV 615) pre-polymer and its cross-linker at ratio of 10:1 was casted on the SU-8 mold (Fig. 2c). After curing at $75^{\circ} \mathrm{C}$ for 2 hours, the PDMS layer was peeled off and treated with TMCS anti-sticking agent. Secondly, the replicated PDMS structure was placed on a glass plate and a solution of polyethylene glycol diacrylate (PEGDA $\mathrm{Mw}=250$, Sigma) mixed with 1v/v\% Irgacure 2959 (Ciba Specialty Chemicals, France) was injected in the free space of the PDMS-glass assembly, followed by UV exposure for $2 \mathrm{~min}$ (Fig. 2d and 2e). Thirdly, a monolayer of gelatin nanofibers was electrospun and cross-linked on the opposite side of the mesh. This has been done by using a similar fabrication protocol of our previous work [19,21]. Briefly, the electrospinning was performed with a gelatin solution of $10 \mathrm{wt} \%$ gelatin powder (G2625, Sigma) in DI water, ethyl acetate, and acetic acid at a volume ratio of $10: 14: 21$. The ejection of the gelatin solution through a needle (23-gauge) to the collector (cage) was controlled with at a speed of $0.2 \mathrm{ml} / \mathrm{h}$, a distance of $10 \mathrm{~cm}$ and bias voltage was $11 \mathrm{kV}$, respectively (Fig. 2f). The sample was then dried overnight in a desiccator and the gelatin nanofibers was soaked in an ethanol solution containing $2 \mathrm{M}$ N-hydroxysuccinimide (NHS, Sigma) and 0.2 M 1ethyl-3-(3-dimethylaminopropyl)carbodiimide hydrochloride(EDC, Sigma) for $4 \mathrm{~h}$. The sample was then washed three times in ethanol and dried in a vacuum overnight. Finally, the nanofiber layer was treated with a solution of agarose (Fisher Scientific, France) $0.2 \mathrm{w} / \mathrm{v} \%$ in DI water and the cage device was dehydrated in oven at $60^{\circ} \mathrm{C}$ for 2 hours.

\subsection{Cell culture}

U87-MG cells (human glioblastoma cells) were grown in T75-flask inside an incubator of $37^{\circ} \mathrm{C}$ and $5 \% \mathrm{CO}_{2}$. The culture medium was Dulbecco's Modified Eagle Medium (DMEM) supplemented with $10 \%$ fetal bovine serum and $1 \%$ penicillin-streptomycin. The cells were passaged using TrypLE express enzyme when they reached $80-90 \%$ confluency. The cells were resuspended in complete DMEM medium and dispersed at density $1.5 \times 10^{5}$ cells per device in the cage through the mesh. Then, the device was placed in the culture dish for incubation under conventional culture conditions for up to 10 days and the medium was exchanged each two days. Prior to cell seeding, the cage devices were sterilized in $70 \%$ ethanol with exposure to UV during $30 \mathrm{~min}$ and equilibrated in complete culture medium for 1 hour.

\subsection{SEM observation}

Caged tumor spheroids were fixed in PBS containing 4\% formaldehyde for 15 minutes. Then, they were rinsed twice with PBS buffer, and merged in 30\% ethanol (in DI water) for $30 \mathrm{~min}$. Afterward, the samples were dehydrated in a graded series of ethanol solutions with concentrations of $50 \%, 70 \%, 80 \%$, 
$90 \%, 95 \%$, and $100 \%$, respectively, and in each for $10 \mathrm{~min}$. Before observation, a $2 \mathrm{~nm}$ thick gold layer was deposited on the samples by sputtering. The images were captured with a SEM machine (Hitachi TM3030) operated at $15 \mathrm{kV}$.

\subsection{Microfluidic culture}

After incubation for 3 days, the device with caged tumor spheroids was reversibly integrated into a microfluidic chip (MesoBioTech, France) and a tangent flow was applied at a flow rate of $0.1 \mathrm{ml} / \mathrm{h}$ in the mesh side using a syringe pump. After incubation for another 3 days, the chip was dismounted and the cage device was placed in a $35 \mathrm{~mm}$ dish with complete culture medium for further observation.

\subsection{Drug test and live/dead assay}

The cage device with tumor spheroids was placed in another culture medium containing $1 \mu \mathrm{M}$ anticancer drug Combretastatin A4 (CA4) [23]. After incubation for 24 hours, disassembling of the tumor spheroids were observed and live/dead assay was performed with PBS containing $4 \mu \mathrm{M}$ Calcein AM (Invitrogen, L3224) and $5 \mu \mathrm{M}$ propidium iodide (Sigma-Aldrich, P4170) solution, respectively. After incubation for $30 \mathrm{~min}$, the cage device was rinsed twice with fresh PBS to remove any residual staining molecules and kept in PBS for observation. Finally, fluorescence images were recorded with an inverted fluorescence microscope (Axio Observer Z1, Zeiss).

\section{Results and discussion}

\subsection{Cage device}

The fabrication process of the cage device is straightforward by using different lithography methods, including UV lithography, soft lithography and micro-aspiration assisted lithography. To study the tumor growth in different size of space, different height 200 (H200), 250 (H250), 300 (H300) $\mu \mathrm{m}$ of cage devices were fabricated as shown in Fig 3a-3c. Fig. 3d and 3e shows respectively the top-view of a microframe with honeycomb compartments with $200 \mu \mathrm{m}$ mesh size and a $1.2 \mu \mathrm{m}$ thick agarose layer with gelatin nanofibers backbone on another side of the frame. Thus, single cells could be introduced on the agarose layer through the mesh and then gave rise to the formation of caged tumor spheroids.

\subsection{Caged tumor spheroids}

Fig 4a shows the tumor spheroids in the cages of different height at day 1, 4, 5, 6. Cell aggregation and spheroid formation could be observed at day 2. At day 6 after cell seeding, caged spheroids were fixed and dehydrated for SEM observation. Despite the decreased spheroid volume after dehydration, remarkably, we observed an important nanofiber deflection underneath of the tumor spheroids as shown in Fig 1c and 1d. Considering the fact that the final tumor size (about $220 \mu \mathrm{m}$ ) is comparable to the height of the cage $(200 \mu \mathrm{m})$, the tumor spheroids trended to keep their spheroid shape by deflecting the 
nanofiber layer without flattening. In addition, uniform formation of tumor spheroid in each large honeycomb can be seen in Fig 1e.

Fig $4 \mathrm{~b}$ shows clearly that the tumor size increased until day 4 and started to decrease at day 5 . This is probably due to the change from single cells to cell aggregations and the strong effect of cell-cell interaction. It is also interestingly to note that the tumor size increased when they were in a larger height of cage device. However, the final size always remained stable in all three heights of cage devices, due probably to the limitation of device height because spheroid size in $\mathrm{H} 200$ and $\mathrm{H} 250$ cage devices both exceeded the cage height. Besides, there could be not enough nutrition diffusion in center area of the spheroids, preventing cell proliferation. The above experiments have been repeated for five times, showing the same results.

\subsection{Microfluidic integration}

The tumor spheroids formed in the cage were stable and could be easily handled and subjected to different stimuli. The cage device was taken out from the dish at day 3 and was integrated into a microfluidic culture device, for another 3 days diffusive culture, as shown in Fig 5a and 5b. The culture medium flowed in the space outside the cage and passed by the honeycomb mesh side at a flow rate of $0.1 \mathrm{ml} / \mathrm{h}$. Figure $4 \mathrm{c}$ shows dead/live image of tumor spheroid cultured in a cage device under cis-flow or diffusive culture conditions, showing the feasibility of culturing them under flow conditions. Clearly, the tumor spheroid could be maintained with few dead cells, and large variety of perturbation can thus be applied.

\subsection{Anti-cancer drug effects}

In order to investigate the drug effect on the spheroids formed in cage devices, combretastatin A4 (CA4) was used for anti-cancer study at day 6 . This drug binds much better to the colchicine site on tubulin to inhibit polymerization and further prevent cell division, thus it is highly cytotoxic to a variety of human cancer cells. As expected, the caged spheroids taken out from a culture dish at day 6 were then immersed for 2 days in $1 \mu \mathrm{M}$ CA4 supplemented culture medium and showed a clear disassembling effect, as shown in Fig 6. The live/dead images showed that there were more dead cells in center area of smaller spheroids, while less in larger spheroids, also due to the drug permeability to the center core, especially in larger height of cage devices. Therefore, these images clearly demonstrated the drug resistance of larger spheroids and strong cancer cell-cell interaction.

Finally, we would like to mention that the deflection of the fiber layer due to the growing tumor spheroid might be used to study the mechanical properties of the tumor. Previously, Stylianopoulos et al have addressed this issue and found that evolution of stress and growth rate both depend strongly on the mechanical interactions with the surrounding host tissue [24, 25]. They suggested that the solid stress on tumor growth involved not only the inhibitory effect of stress on cancer cell proliferation and the 
induction of apoptosis, but also the resistance of the surrounding tissue to tumor expansion. By studying the mechanic interaction of stem cell colonies with a 3D patterned elastomeric substrate, a contractile force of the order of $1 \mathrm{mN}$ was deduced. In the present case, the solid stress causing the deflection of the fiber layer can be estimated by considering an edge-clamped thin disk. Based on the theory of plates and shells [26], the pressure $\Delta \mathrm{p}$ on the disk is given by

$$
\Delta p=\frac{16 E h^{3}}{3\left(1-v^{2}\right) R^{4}}\left(\delta+0.488 \frac{\delta^{3}}{h^{2}}\right)
$$

where $h$ and $R$ and the thickness and radius of the disk, $E$ and $v$ are the Young's module and Poisson ratio of disk material, $\delta$ is the deflection of the disk center point. Assuming a disk of $0.1 \mu \mathrm{m}$ thickness and $50 \mu \mathrm{m}$ radius with a Young module of $10 \mathrm{MPa}$ and a Poison ratio $<0.3$, a pressure of $\sim 3.4 \mathrm{kPa}$ is obtained, which is in the range of previous findings [25]. More detailed analyses are needed for quantitative assessments.

\section{Conclusions}

We have developed a fabricated method to produce caged tumor spheroids. This cage device is robust and easy to use. Uniform tumor spheroids could be generated in the cages by single cell seeding and static culture. If the height of the cage is too small, the underneath fibers were deflected due to mechanical stress of the growing tumors. If the height of the case is too large, tumors grew freely and their size was limited by diffusion. The caged tumor spheroids were also integrated in a microfluidic chip and they could be cultivated under tangent flow conditions but dissembled after drug introduction. More systematic studies will be carried out by considering other types of stress such as hypoxia, ischemia, compression, etc. and isolation of the released cells [22], thereby faciliating in-vitro cancer modeling.

\section{Acknowledgements}

This work was supported by Agence de Recherche Nationale under contract ANR-17-CE09-0017 (AlveolusMimics), European Commission Cost Action BIONECA (CA 16122), DIM ELICIT program of Ile-de-France, and PSL Valorization through Pre-maturation project. Yong He is grateful to the China Scholarship Council for grant of his $\mathrm{PhD}$ studies. 


\section{References}

[1] F. Bray, J. Ferlay, I. Soerjomataram, R.L. Siegel, L.A. Torre, A. Jemal, Global cancer statistics 2018: GLOBOCAN estimates of incidence and mortality worldwide for 36 cancers in 185 countries, CA: a cancer journal for clinicians, 68(6) (2018) 394-424.

[2] G.B.o.D.C. Collaboration, Global, regional, and national cancer incidence, mortality, years of life lost, years lived with disability, and disability-adjusted life-years for 29 cancer groups, 1990 to 2016: a systematic analysis for the global burden of disease study, JAMA oncology, 4(11) (2018) 1553-1568. [3] T. Rodrigues, B. Kundu, J. Silva-Correia, S.C. Kundu, J.M. Oliveira, R.L. Reis, V.M. Correlo, Emerging tumor spheroids technologies for 3D in vitro cancer modeling, Pharmacology therapeutics, 184 (2018) 201-211.

[4] M.E. Katt, A.L. Placone, A.D. Wong, Z.S. Xu, P.C. Searson, In vitro tumor models: advantages, disadvantages, variables, and selecting the right platform, Frontiers in bioengineering and biotechnology, 4 (2016) 12.

[5] E.C. Costa, A.F. Moreira, D. de Melo-Diogo, V.M. Gaspar, M.P. Carvalho, I.J. Correia, 3D tumor spheroids: an overview on the tools and techniques used for their analysis, Biotechnology advances, 34(8) (2016) 1427-1441.

[6] Y.-C. Tung, A.Y. Hsiao, S.G. Allen, Y.-s. Torisawa, M. Ho, S. Takayama, High-throughput 3D spheroid culture and drug testing using a 384 hanging drop array, Analyst, 136(3) (2011) 473-478.

[7] R. Foty, A simple hanging drop cell culture protocol for generation of 3D spheroids, JoVE, (51) (2011) e2720.

[8] T.-M. Achilli, J. Meyer, J.R. Morgan, Advances in the formation, use and understanding of multicellular spheroids, Expert opinion on biological therapy, 12(10) (2012) 1347-1360.

[9] D. Loessner, K.S. Stok, M.P. Lutolf, D.W. Hutmacher, J.A. Clements, S.C. Rizzi, Bioengineered 3D platform to explore cell-ECM interactions and drug resistance of epithelial ovarian cancer cells, Biomaterials, 31(32) (2010) 8494-8506.

[10] F. Ozawa, K. Ino, T. Arai, J. Ramón-Azcón, Y. Takahashi, H. Shiku, T. Matsue, Alginate gel microwell arrays using electrodeposition for three-dimensional cell culture, Lab on a Chip, 13(15) (2013) 3128-3135.

[11] Y.-s. Torisawa, A. Takagi, Y. Nashimoto, T. Yasukawa, H. Shiku, T. Matsue, A multicellular spheroid array to realize spheroid formation, culture, and viability assay on a chip, Biomaterials, 28(3) (2007) 559-566.

[12] I. Martin, D. Wendt, M. Heberer, The role of bioreactors in tissue engineering, TRENDS in Biotechnology, 22(2) (2004) 80-86.

[13] R.Z. Lin, H.Y. Chang, Recent advances in three-dimensional multicellular spheroid culture for biomedical research, Biotechnology Journal: Healthcare Nutrition Technology, 3(9 - 10) (2008) 11721184. 
[14] G.R. Souza, J.R. Molina, R.M. Raphael, M.G. Ozawa, D.J. Stark, C.S. Levin, L.F. Bronk, J.S. Ananta, J. Mandelin, M.-M. Georgescu, Three-dimensional tissue culture based on magnetic cell levitation, Nature nanotechnology, 5(4) (2010) 291.

[15] W.L. Haisler, D.M. Timm, J.A. Gage, H. Tseng, T. Killian, G.R. Souza, Three-dimensional cell culturing by magnetic levitation, Nature protocols, 8(10) (2013) 1940.

[16] J.M. Karp, J. Yeh, G. Eng, J. Fukuda, J. Blumling, K.-Y. Suh, J. Cheng, A. Mahdavi, J. Borenstein, R. Langer, Controlling size, shape and homogeneity of embryoid bodies using poly (ethylene glycol) microwells, Lab on a Chip, 7(6) (2007) 786-794.

[17] Y. Tang, J. Liu, Y. Chen, Agarose multi-wells for tumour spheroid formation and anti-cancer drug test, Microelectronic Engineering, 158 (2016) 41-45.

[18] F. Mirab, Y.J. Kang, S. Majd, Preparation and characterization of size-controlled glioma spheroids using agarose hydrogel microwells, PloS one, 14(1) (2019) e0211078.

[19] Y. Tang, L. Liu, J. Li, L. Yu, L. Wang, J. Shi, Y. Chen, Induction and differentiation of human induced pluripotent stem cells into functional cardiomyocytes on a compartmented monolayer of gelatin nanofibers, Nanoscale, 8(30) (2016) 14530-14540.

[20] Y. Tang, F.P.U. Severino, F. Iseppon, V. Torre, Y. Chen, Patch method for culture of primary hippocampal neurons, Microelectronic Engineering, 175 (2017) 61-66.

[21] Y. Tang, L. Liu, J. Li, L. Yu, F.P.U. Severino, L. Wang, J. Shi, X. Tu, V. Torre, Y. Chen, Effective motor neuron differentiation of hiPSCs on a patch made of crosslinked monolayer gelatin nanofibers, Journal of Materials Chemistry B, 4(19) (2016) 3305-3312.

[22] Y. Tang, J. Shi, S. Li, L. Wang, Y.E. Cayre, Y. Chen, Microfluidic device with integrated microfilter of conical-shaped holes for high efficiency and high purity capture of circulating tumor cells, Scientific Reports, 4 (2014) 6052.

[23] G.C. Tron, T. Pirali, G. Sorba, F. Pagliai, S. Busacca, A.A. Genazzani, Medicinal chemistry of combretastatin A4: present and future directions, Journal of medicinal chemistry, 49(11) (2006) 3033 3044.

[24] C. Voutouri, F. Mpekris, P. Papageorgis, A.D. Odysseos, T. Stylianopoulos, Role of constitutive behavior and tumor-host mechanical interactions in the state of stress and growth of solid tumors, PloS one, Frontiers in oncology, 9(8) (2014) e104717.

[25] M. Kalli, T. Stylianopoulos, Defining the role of solid stress and matrix stiffness in cancer cell proliferation and metastasis, Frontiers in oncology, 8 (2018) 55.

[26] S.P. Timoshenko, S. Woinowsky-Krieger, Theory of plates and shells, McGraw-hill1959. 


\section{Figure caption}

Figure 1. (a) Schematic of a cage, caged tumor spheroid formation, and tumor growth induced deformation of the underneath nanofiber monolayer; (b) Photograph of a cage device; (c-d) Scanning electron microscopy image of a caged tumor spheroid and tumor growth induced deflection of the nanofiber monolayer; (e) Microphotograph of caged tumor spheroids in each of the honeycomb compartments.

Figure 2. Schematic diagram of the fabrication process of the cage device: (a) Cage layer patterning of the mold by UV lithography and SU8 resist on a silicon wafer; (b) Microframe patterning of the mold by UV lithography and another SU8 resist layer; (c) Replication of the mold pattern into PDMS by soft lithography; (d) Secondary replica of the mold patterning by micro-aspiration assisted UV curing of PEGDA; (e) Microframe with mesh structures released from glass substrate; (f) Electrospinning, crosslinking and agarose coating of the gelatin nanofiber layer.

Figure 3. Scanning electron microscopy images of the fabricated cages: Sideview of the cages with height $200 \mu \mathrm{m}$ (a, H200), $250 \mu \mathrm{m}$ (b, H250) and $300 \mu \mathrm{m}$ (c, H300), respectively; (d) Top-view of the mesh of hole-size $200 \mu \mathrm{m}$ (d) and agarose coating nanofibers (e).

Figure 4. Cage height and incubation time dependences of the caged tumor spheroids: (a) Microphotograph of tumor spheroids in H200, H250 and H300 cages at day 1, 4, 5, 6; (b) Size variation of the spheroids in different cages up to 6 days.

Figure 5. Schematic (a) and photograph (b) of a microfluidic chip for the cage device integration; (c) Live/dead fluorescence images of tumor spheroids after incubation for 3 days in a H200 cage device.

Figure 6. Bright field and live/dead fluorescence images of tumor spheroids confined in H200, H250 and H300 cages after days in CA4 supplemented medium (day 6-day 8). 
Figure 1
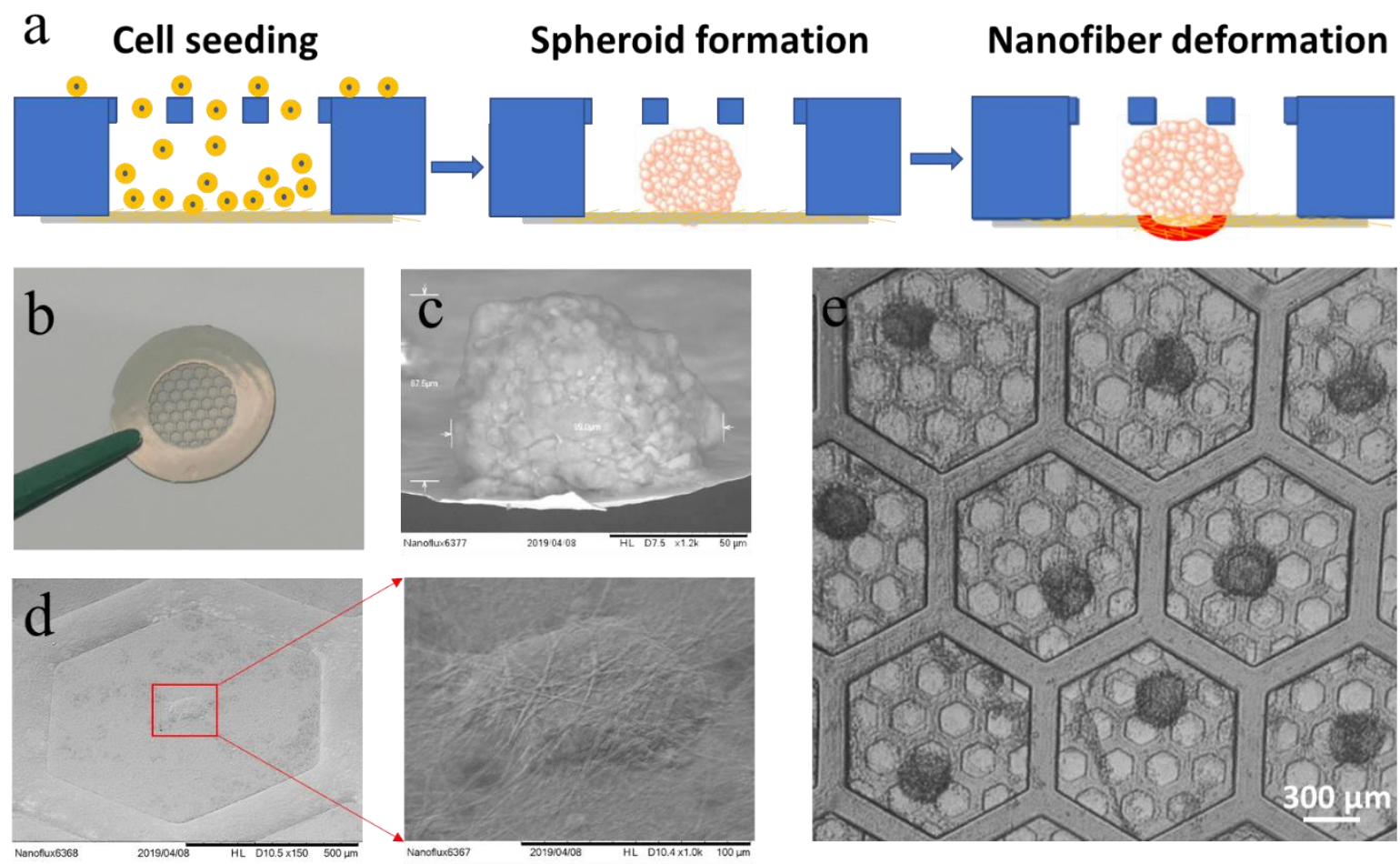

Figure 2

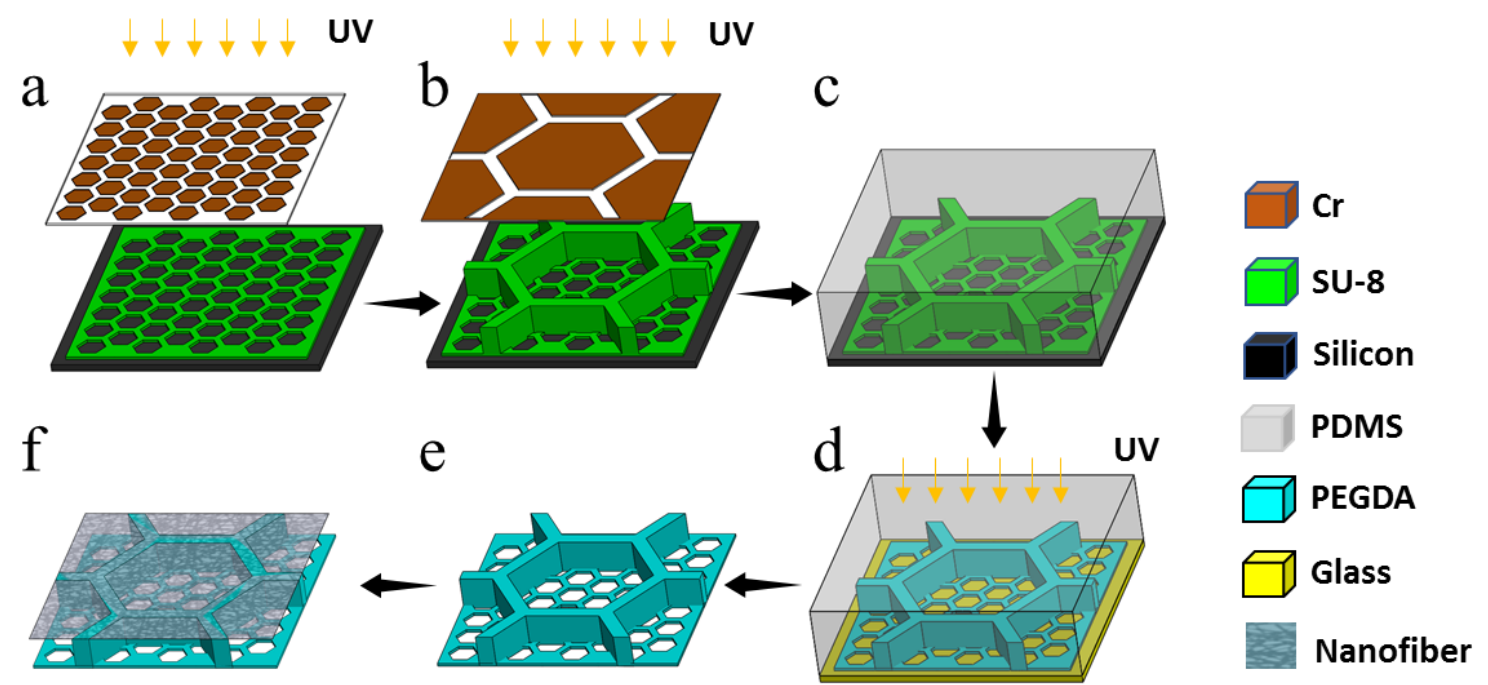


Figure 3
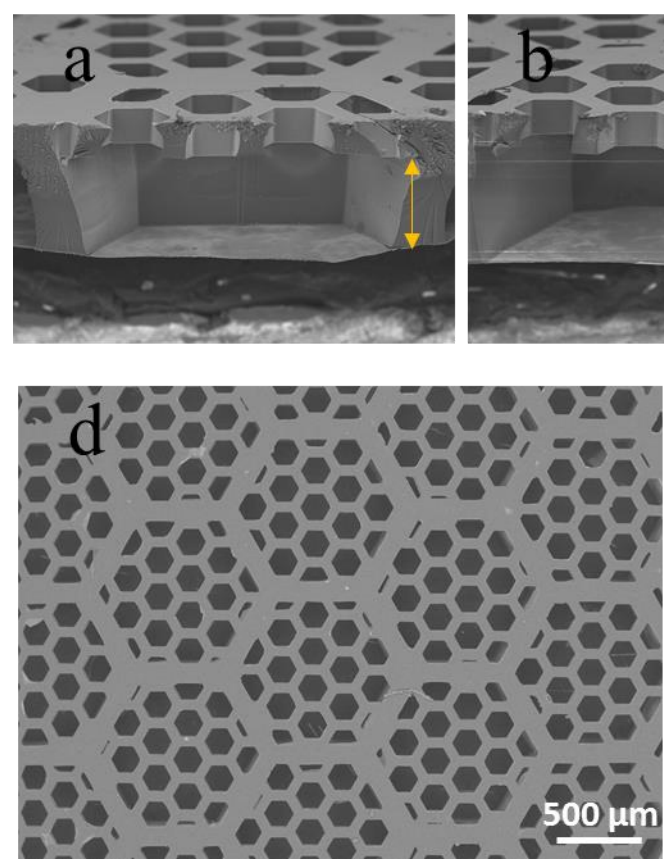
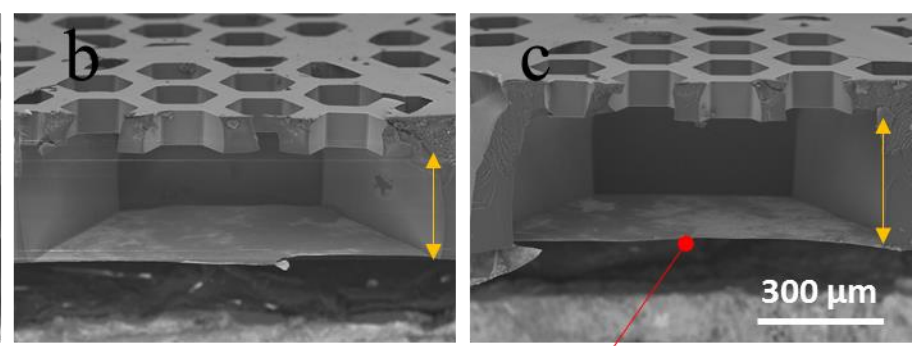

e

$5 \mu \mathrm{m}$

Figure 4
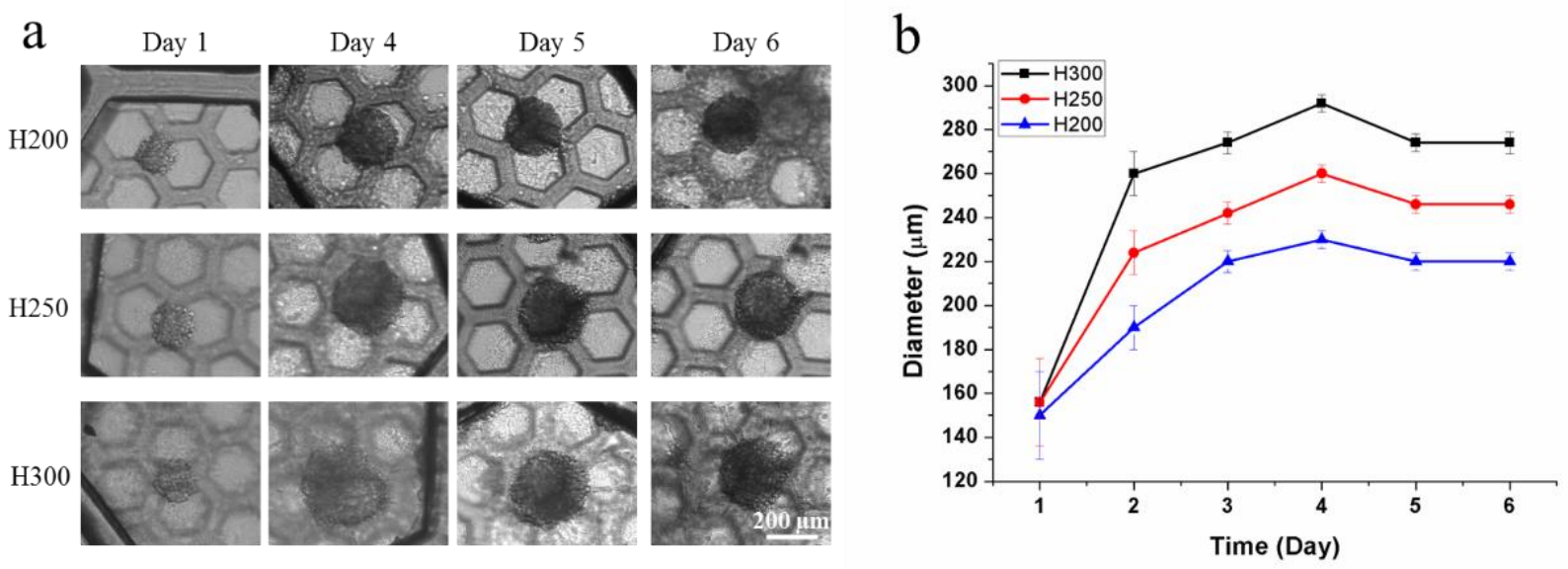
Figure 5

a

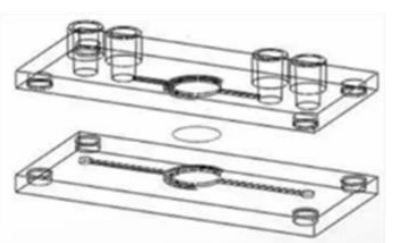

b

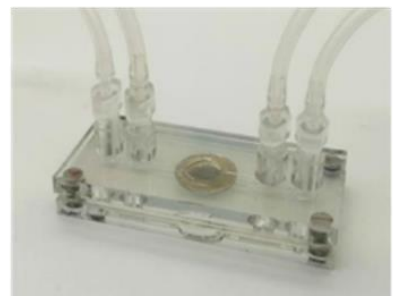

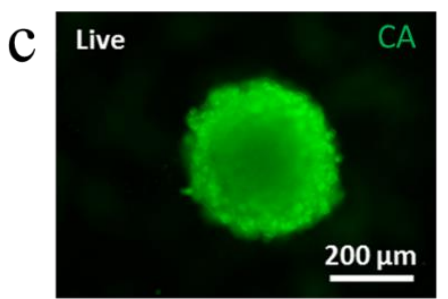

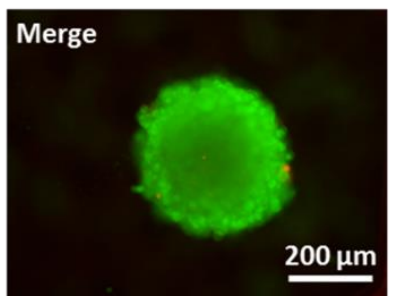

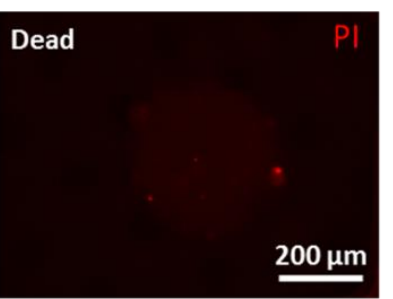

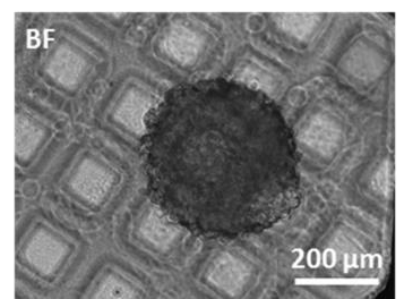

Figure 6
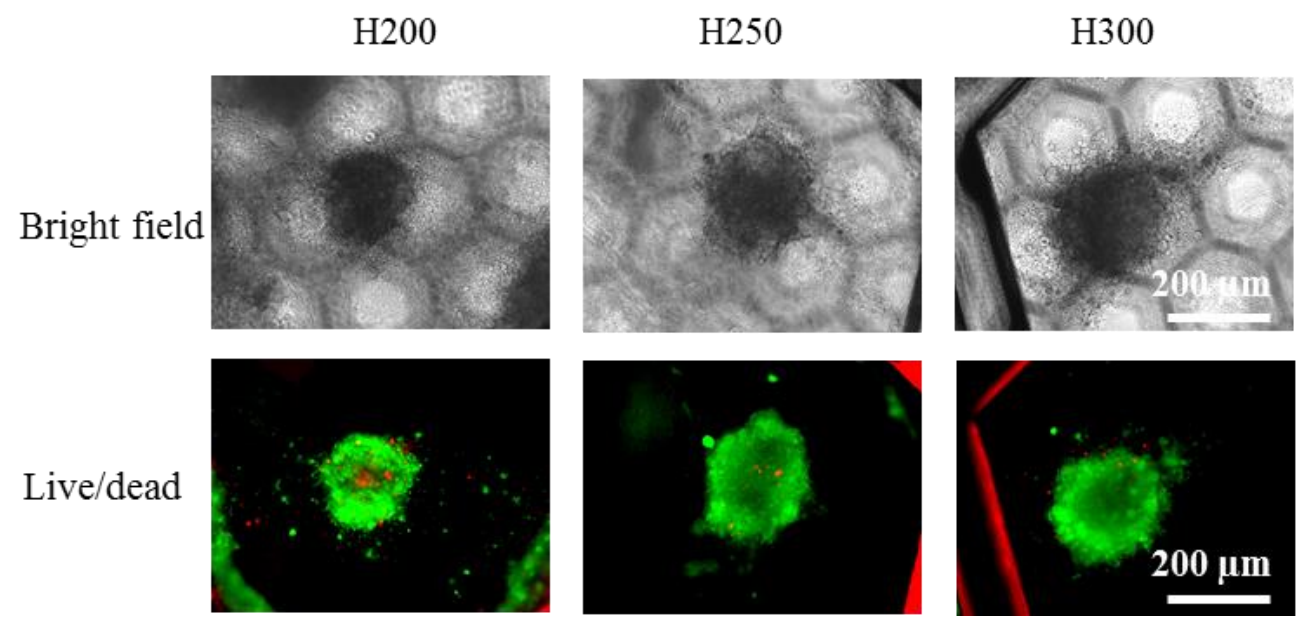